\title{
Analysis of the Negative Impact of Hydraulic Fracturing Technology on the Environment
}

\author{
Vitaly Zhironkin ${ }^{1, *}$, and Michal Cehlar ${ }^{2}$ \\ ${ }^{1}$ National Research Tomsk Polytechnic University, 634050, 30 Lenina st., Tomsk, Russia \\ ${ }^{2}$ Technical University in Kosice, Institute of Earth Resources, Faculty of Mining, Ecology, Process \\ Control and Geotechnologies, Park Komenského 19, 042000 Košice, Slovakia
}

\begin{abstract}
The development of the oil and gas industry around the world is connected with the development of unconventional fields, such as shale oil, using modern technologies, for example, rotary steerable systems, which allow more accurate and high-quality work on well construction, as well as multi-stage hydraulic fracturing, allowing more efficient extracting of hydrocarbons from the deposits. However, like any mining of minerals, the extraction of hydrocarbon raw materials has a harmful effect on the ecology of the area in which the extraction takes place. This occurs due to the use of a large number of chemical compounds, a small proportion of processing of rocks obtained during drilling, cleaning up territories to create infrastructure for organizing the extraction work of raw materials. The introduction of new fields into development leads to the increase in the level of industrial pollution of the environment. The impact of hydraulic fracturing technology on the environmental situation in the field is considered in the article. The author analyzes the impact of this method of increasing oil development on the environment, the experience of using this technology abroad, as well as government regulation of the use of this technology by producing companies around the world.
\end{abstract}

\section{Introduction}

In the oil production industry, environmental pollution begins with exploratory drilling and construction of oil and gas production wells. The main sources of pollution during well construction are diesel exhaust from drilling rigs, mud degassers, storage tanks for bulk powder materials, sludge pits with industrial and technological waste, as well as circulation systems. In many cases, drilling rigs do not take measures to protect soil and water bodies from pollution. As a result, the adjacent lands are contaminated with drilling mud and oil products. Then these harmful substances are washed off by melt water onto the adjacent lands and water bodies. The process of soil pollution is aggravated by the presence of highly mineralized strata and waste waters in the soil, which are pumped to maintain pressure in productive strata and absorbing horizons. Within a radius of 500-800 $\mathrm{m}$ from the drilling rig, vegetation is destroyed by $70-80 \%$, and within a radius of $100 \mathrm{~m}$, as a result of more intense mud pollution, it practically disappears. Industrial drilling waste accumulates in dumps at the

\footnotetext{
* Corresponding author: v.zhironkin@inbox.ru
} 
drilling site and requires subsequent evacuation and placement in a specially designated area for the sludge storage. At the same time, the issues of assessing the polluting capacity of waste are of particular importance [1]. The amount and toxicity of pollutants in groundwater depend on this. The main and most potent contaminant in drilling operations is drilling mud. For its preparation, up to two dozen chemical reagents are used.

However, according to domestic ecologists [2-4], as well as foreign scientists [5, 6], one of the most harmful measures in oil and gas production is hydraulic fracturing technology. Hydraulic fracturing is a process that involves the introduction of a mixture of water, sand and chemicals into gas-bearing rocks under high pressure (500-1500 atm) (Fig. 1). This leads to the formation of small fractures, which improve the reservoir properties in the bottomhole zone, which leads to a significant increase in the well rate. All this system of fractures connects the well with the productive parts of the formation remote from the bottomhole. To prevent the closure of cracks after pressure reduction, coarse sand is introduced into them, added to the fluid injected into the well. The radius of cracks can reach several tens of meters.

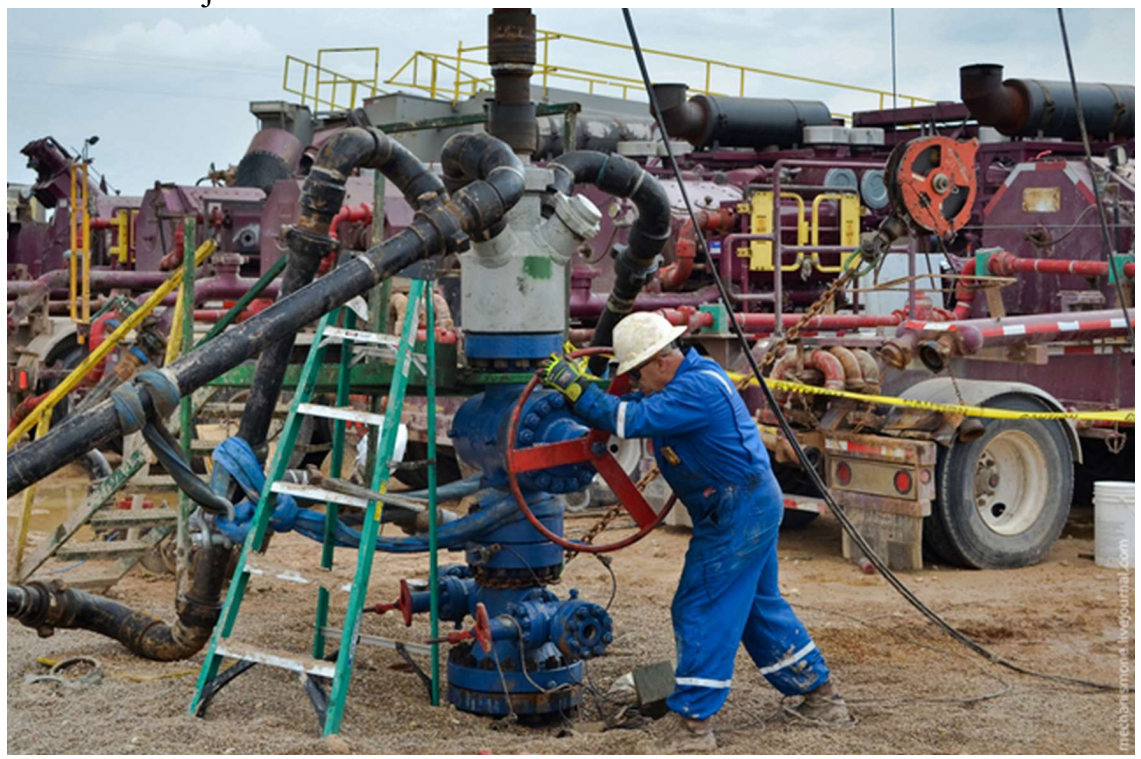

Fig. 1. Installation for hydraulic fracturing.

\section{Materials and Methods}

The main goal of the hydraulic fracturing method is to increase the permeability of the treated near-wellbore (bottomhole) formation zone by creating artificial fractures and expanding natural fractures in the formation, as well as increasing the well rate, "revitalizing" idle and unprofitable wells.

The main environmental hazard in shale gas production is the use of large amount of chemicals that mix with water and sand. The operation of hydraulic fracturing in one area has to be repeated up to 10 times a year. During hydraulic fracturing, the chemical mixture permeates the rock, which leads to the pollution of large areas, as well as groundwater. (Fig. 2). Hydraulic fracturing causes irreparable damage to the environment. According to the United States Environmental Protection Agency, there are a number of environmental problems associated with the use of hydraulic fracturing [7]. There have been cases when hydraulic fracturing led to the appearance of methane and many impurities harmful to humans, including benzene, toluene, ethylbenzene, in water from an artesian well located in a settlement relatively close to the field. This is because 80 to 300 tons of chemicals are used 
for each fracturing operation. According to ecologists, in places where hydraulic fracturing is used, water becomes unsuitable for drinking, people get sick more often, animals lose their hair, and air quality deteriorates.

The use of hydraulic fracturing technology in the production of shale gas and oil can cause very serious environmental problems in the production region, which can affect the life and health of people both working in the field and living nearby [8]. The impact can be exerted not only on groundwater, but also on seismic activity. In addition, according to the research conducted by the American scientists, the production of shale gas by hydraulic fracturing led to the earthquakes in Ohio in 2013.

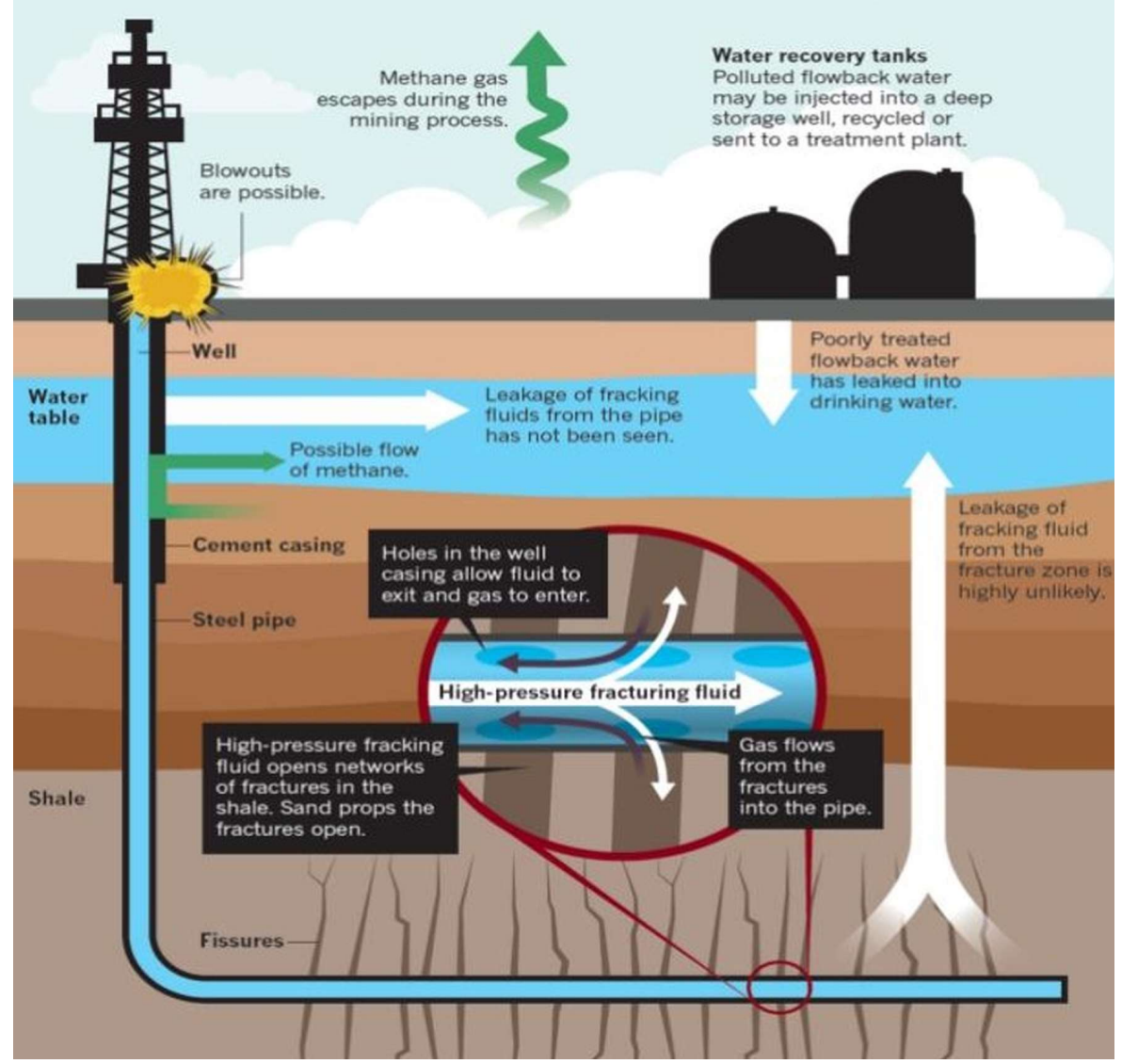

Fig. 2. The principal scheme of hydraulic fracturing.

It should be noted that in terms of technology, hydraulic fracturing has become a real breakthrough, which has significantly increased the well rates at the late stage of production. This is due to the fact that hydraulic fracturing increases the oil recovery efficiency - a technological parameter of field development, showing how much fluid comes to the surface from the total volume in the reservoir. As the oil recovery efficiency increases that allows extracting more oil from one well, instead of drilling new ones for additional development of the given shelf. In addition, this technology opened the way for the development of oil from unconventional reservoirs, which led to the so-called shale revolution in the United States and some other countries. Therefore, at old fields in Russia, such as Ust Balykskoye and Mamontova, the use of hydraulic fracturing gave an opportunity to increase the current production, prevent the decline that had been already planned, and in many aspects revive the old fund. However, Satellite Observations have detected gigantic emissions of methane 
into the atmosphere in the shale gas production area in the United States - previously, these emissions were greatly underestimated. According to a widespread theory, it is believed that the increase in methane in the atmosphere is the cause of the greenhouse effect and global warming.

Technologically, hydraulic fracturing is associated with the circulation of a large amount of a mixture of water, chemicals, surfactants and sand [9]. Therefore, near the deposits there is a significant accumulation of waste water contaminated with chemicals, which can get into the soil, destroying its fertile properties and groundwater. After pumping water into the well under high pressure, the waste liquid is partially pumped out to the surface and stored in tanks, and then it must be cleaned. This water is evaporated, which also pollutes the air, but not all water is purified from chemical pollution. Part of the waste water is re-saturated with chemicals and reused for subsequent hydraulic fracturing stages, less often it is purified at special plants. Among other things, the production of shale gas by hydraulic fracturing leads to the pollution of groundwater with benzene, toluene, dimethylbenzene, ethylbenzene, arsenic and many other hazardous substances.

One hydraulic fracturing operation requires 80-300 tons of chemicals up to 500 names. The damage from shale gas extraction by this method is so great that one hydraulic fracturing is enough for the groundwater to acquire a metallic taste, inappropriate color and become hazardous to use. So in some mining areas, the water in the wells can be set on fire. Such contamination of the fertile layer and groundwater turns the land area into a desert for several years. There is also a possibility that the gas that could not be pumped out can react with chemicals that are pumped into the soil by technology and seeps through cracks to the surface. When shale gas is extracted, significant methane losses of 3.6-7.9\% occur, which leads to an increase in the greenhouse effect. The level of greenhouse gas emissions from the mining process is significantly higher than from the extraction of other types of fossil fuels. Also, there is a high probability of contaminating the industrial water with radioactive substances, which are subsequently carried to the surface together with the water.

Many oil companies, including manufacturers in the United States and abroad, note the environmental hazard of hydraulic fracturing technology, as well as the risks of destructive processes in the soil itself. They include seismic instability and earthquakes, as well as soil subsidence, which can be more than ten meters. In dry areas or areas without water bodies nearby, hydraulic fracturing water is extracted from the ground, which creates additional voids underground. The harm from the production of shale gas or oil using hydraulic fracturing technology can significantly reduce the life quality in nearby settlements due to the deterioration of the environmental situation in the area. In places where hydraulic fracturing is used, the water becomes unsuitable for drinking, which immediately affects human health, there is an increase in cancer and lung diseases as well. It occurs because the water used during hydraulic fracturing can remain on the surface for a long time, as a result, there is a high level of gamma radiation, animals lose their hair, and air quality deteriorates.

The practice of the USA has shown that despite the fact that hydraulic fracturing is carried out much below the level of foot water, the soil layer, groundwater and air can be contaminated with toxic substances and methane. Frequent fracturing leads to an increase in the permeability of the rocks, and through the cracks, chemicals and methane seep upward.

In the United States, the authorities of the states Vermont (2012) and New York (December 2014) banned gas production by hydraulic fracturing on their territory [10]. A considerable number of countries and even states in the US have introduced a moratorium on the production of shale oil and gas on their territory by hydraulic fracturing. In April 2014, an American family from Texas won the first ever case on negative effects of shale gas production by hydraulic fracturing. The family received compensation from the oil company "Aruba Petroleum" for contaminating their site (including a well with water that became undrinkable) and damaging their health. The use of hydraulic fracturing was banned by the 
Bulgarian parliament in January 2012. In September 2013, the Dutch government introduced a temporary ban on the use of hydraulic fracturing technology for gas production. In December 2014, the government of Mark Rutte passed a resolution to extend the ban on the use of hydraulic fracturing technology in the Netherlands until 2016. In June 2015, the European Union court demanded to carry out an environmental review before each explorational drilling in order to extract hydrocarbons. At the same time, a study in which the US Geological Survey and the University of Colorado at Boulder confirmed the connection between fracturing and earthquakes was published. In June 2015, the Court of the European Union ordered to conduct an environmental expertise prior to each explorational drilling in order to extract hydrocarbons.

\section{Results}

The main environmental problem, according to many experts, is the possibility of contaminating water bearing formations with methane and used solutions. It is considered that even a purified drilling mud, in which chemical additives account for only $1-2 \%$, is capable of poisoning groundwater, the more so as during hydraulic fracturing, only a third of the water used is raised from wells. Some components that are used to achieve the required viscosity of the fracturing fluid are carcinogenic; therefore, their penetration into formations containing groundwater is dangerous. In addition, fractures from hydraulic fracturing can move upward, contaminating the pound water with the injected fluid or contributing to the release of methane. In the result of fracturing, radiation enters the upper layers of sedimentary rocks, as well as to the surface. In addition, water pumped out from depths of more than 2 $\mathrm{km}$ is saturated with radioactive elements. An increase in radiation inevitably leads to an increase in serious diseases among workers, as well as among local residents living near the fracturing zone. The seismic activity risks are also inevitable. The cases when earth shocks were recorded in places of oil production by hydraulic fracturing are known. They can be caused by the numerous hydro blows, and as a result of the seepage of water residues from wells into cracks, erosion of rocks and an increase in their mobility.

The existing environmental problems in at least three states of the US are gradually acquiring the status of a disaster. It is for this reason that shale gas has been banned in NewYork State. In Europe, according to many experts, the production of shale gas will not be widely developed, since the environmental legislation is stronger than in the United States. The main reason why the hydraulic fracturing method still finds its application is a sharp increase in the well flow rate during operation. The method allows "reviving" idle wells, where the extraction of mineral resources by traditional methods is unprofitable or even impossible. However, are the shale gas extraction and the method itself so beneficial? Despite the fact that at present it is capable of bringing large profits to oil companies, in the future, a large amount of money can be spent on rebuilding the destroyed environment. Still, financial losses cannot be compared with the huge irreparable harm that the hydraulic fracturing method can cause to the environment and, accordingly, to human life and health.

\section{Conclusion}

Hydraulic fracturing as a method for intensifying oil production causes a lot of controversy and disagreements among specialists in the field of geology and ecology. The harm and environmental damage from this technology should not be underestimated, however, many aspects of this technology impact on the environment have long remained outside the scientists' research, however, due to the presence of a long-term effect on the environment, its impact should be studied more thoroughly. The efficiency from using hydraulic fracturing 
technology is undoubtedly high, as well as the profitability; therefore, the reduction in the use of frack in the near future is unlikely, unless a ban on its use in a specific area is imposed. Today in Russia, hydraulic fracturing is actively used in the production of shale hydrocarbons, which makes further research of the environmental issues of the fields where this technology is applied especially relevant. The development of technologies does not stand still, therefore, in the future there are prospects for the creation of less environmentally harmful compounds for hydraulic fracturing. That will make it possible to use the advantages of the technology in full even in the areas that are relatively close to settlements.

\section{References}

1. V. I. Pavlenko, E. K. Glukhareva, S.Yu Kutsenko, Ecological Safety of Oil and Gas Deposits Development on Arctic Shelf of Russia (The 26th International Ocean and Polar Engineering Conference, Rhodes, 2016)

2. L.V. Eder, I.V. Filimonova, I.V. Provornaya, V.Yu. Nemov, Drilling and Oil, 4, 23, (2014)

3. L.V. Kazakova, A. A. Mokrushin, V. F. Khmelev, Drilling and Oil, 3, 18 (2014)

4. A.E. Lukin, The Nature of Shale Gas in the Context of Problems of Oil and Gas Lithology (Nedra, Moscow, 2011)

5. D. Campin, SPE Production and Operations, 30(04), 329-361 (2015)

6. R. Beckwith, Journal of Petroleum Technology, 63(07), 37-40 (2011)

7. U.S. EPA, Hydraulic Fracturing for Oil and Gas: Impacts from the Hydraulic Fracturing Water Cycle on Drinking Water Resources in the United States (U.S. Environmental Protection Agency, Washington, 2016)

8. J.L. Adgate, B.D. Goldstein, L.M. McKenzie, Environment Science Technology, 48(15), 8307-8320 (2014)

9. M.A. AlKhowaildi, M.A. AlGhazal, Al-Driweesh, H. Abdrabalnabi, Eco-Friendly Hydraulic Fracturing Fluid: Field Deployment and Performance Evaluation in Saudi Arabia's Tight Gas Reservoirs (SPE Asia Pacific Hydraulic Fracturing Conference, Beijing, 2016)

10. New York Bans Hydraulic Fracturing (National Law Review, Washington, 2016) 